\title{
Traveling waves and global oscillations triggered by attractive molecular interactions in an excitable system
}

\author{
Karin John, ${ }^{1,2, *}$ Sergio Alonso, ${ }^{3, \dagger}$ and Markus Bär ${ }^{3, \ddagger}$ \\ ${ }^{1}$ Université Grenoble Alpes, LIPhy, F-38000 Grenoble, France \\ ${ }^{2}$ Centre National de la Recherche Scientifique, LIPhy, F-38000 Grenoble, France \\ ${ }^{3}$ Physikalisch-Technische Bundesanstalt, Abbestrasse 2-12, D-10587 Berlin, Germany
}

(Received 13 August 2014; published 17 November 2014)

\begin{abstract}
During pattern formation in spatially extended systems, different mechanisms with different characteristic length scales, e.g., reaction-diffusion processes or molecular interactions, can be active. Such multiscale effects may generate new phenomena, which are not observed in systems where pattern formation occurs on a single scale. Here, we derive and analyze a reaction-diffusion model of the FitzHugh-Nagumo type with short-range attractive molecular interactions of the activator species. The model exhibits a wave instability. Simulations in one and two dimensions show traveling waves with a wavelength set by the parameters of the molecular interaction in the model. In two dimensions, simulations reveal a labyrinthine arrangement of the waves in systems with isotropic diffusion, whereas parallel bands of counterpropagating waves are formed in simulations of a model with anisotropic diffusion. The latter findings are in good qualitative agreement with experimental observation in the catalytic $\mathrm{NO}+\mathrm{H}_{2}$ reaction on an anisotropic $\mathrm{Rh}(110)$ surface. In addition we have identified a transition regime in the simulations, where a short scale instability triggers global oscillations in an excitable regime.
\end{abstract}

DOI: 10.1103/PhysRevE.90.052913

PACS number(s): 82.40.Bj, 82.40.Np, 89.75.Kd

\section{INTRODUCTION}

Formation of spatiotemporal patterns in reaction-diffusion systems is a common feature of self-organized chemical [1] and biological media [2]. Static (e.g., Turing patterns in activator-inhibitor systems) and dynamics structures (e.g., traveling waves in excitable media) are obtained in such nonlinear systems outside of equilibrium. Typical time scales together with the particular values of the diffusion coefficients fix the characteristic spatial scales of both static and dynamic patterns. On the other hand, attractive or repulsive molecular interactions produce additional fluxes, which may induce an accumulation of molecules and can eventually lead to phase separation $[3,4]$. The thereby formed spatial structures result from a relaxation process toward equilibrium.

The cooperative action of chemical reactions, diffusion processes, and molecular interactions has been shown to lead to the formation of complex static and dynamic structures. For example, a theoretical model for phase separating binary mixtures which undergo a simple conversion reaction with linear kinetics [5] revealed stationary stable structures at a finite wavelength resembling a Turing pattern. The same phenomenon has been observed in a model for a phase separating layer of adsorbing and desorbing molecules on a surface [6]. Similarly, experiments and theoretical models on the catalytic water formation on a $\mathrm{Rh}(110)$ surface in the presence of alkali metals as promotors (i.e., chemical species that are not consumed during the reaction but influence the reaction rate locally and might attract or repel certain reactants $[7,8]$ ) showed a slowing down of the reaction fronts [9] and the formation of stationary periodic patterns [10-12].

\footnotetext{
*karin.john@ujf-grenoble.fr

${ }^{\dagger}$ sergio.alonso@ptb.de

${ }^{\ddagger}$ markus.baer@ptb.de
}

Further theoretical studies of reaction-diffusion patterns affected by molecular interactions have predicted traveling structures in a system of mutually attractive reactive adsorbates [13] and in reactive ternary mixtures [14], stationary structures and standing waves in surface reactions coupled to structural phase transitions in the substrate $[15,16]$, as well as stationary patterns and traveling waves in reactive Langmuir layers [17] produced by photoexcitation [18,19] or by transmembrane flows [20,21]. Especially the lipid membranes of living cells are an important system, where molecules are in close proximity and thus phase separation is plausible [22]. At the same time, reaction-diffusion processes take place, couple to the phase separation, and produce complex stationary and dynamical spatial structures [5,23-30].

Another interesting phenomenon has been observed recently experimentally in the $\mathrm{NO}+\mathrm{H}_{2}$ reaction on a $\mathrm{Rh}(110)$ surface. Like many catalytic surface reactions it displays a rich spatiotemporal behavior [32-34], including monostable, bistable, and excitable dynamics, depending on the experimental conditions [35,36]. However, the addition of potassium as a promotor induces the formation of new types of chemical patterns $[31,37,38]$. For example, in the excitable regime one can observe target patterns [see Fig. 1(a)]. Upon addition of potassium to the reaction, the aspect of the pacemaker rapidly changes [see Fig. 1(b)] and eventually short scale traveling waves take over the pacemaker [see Figs. 1(c) and 1(d)]. The velocity of the waves decreases from 1-2 $\mu \mathrm{m} / \mathrm{s}$ without potassium to $0.17 \mu \mathrm{m} / \mathrm{s}$ in the presence of potassium [31].

Motivated by the last example, we study here the effect of attractive molecular interactions on the dynamics of an excitable system of the FitzHugh-Nagumo type. In our analysis we are not predominantly interested in the microscopic details of a specific surface reaction; instead we are interested in investigating the general effect of a Cahn-Hilliard type diffusion term on the dynamical behavior of the excitable system. However, we also compare the results of the model 

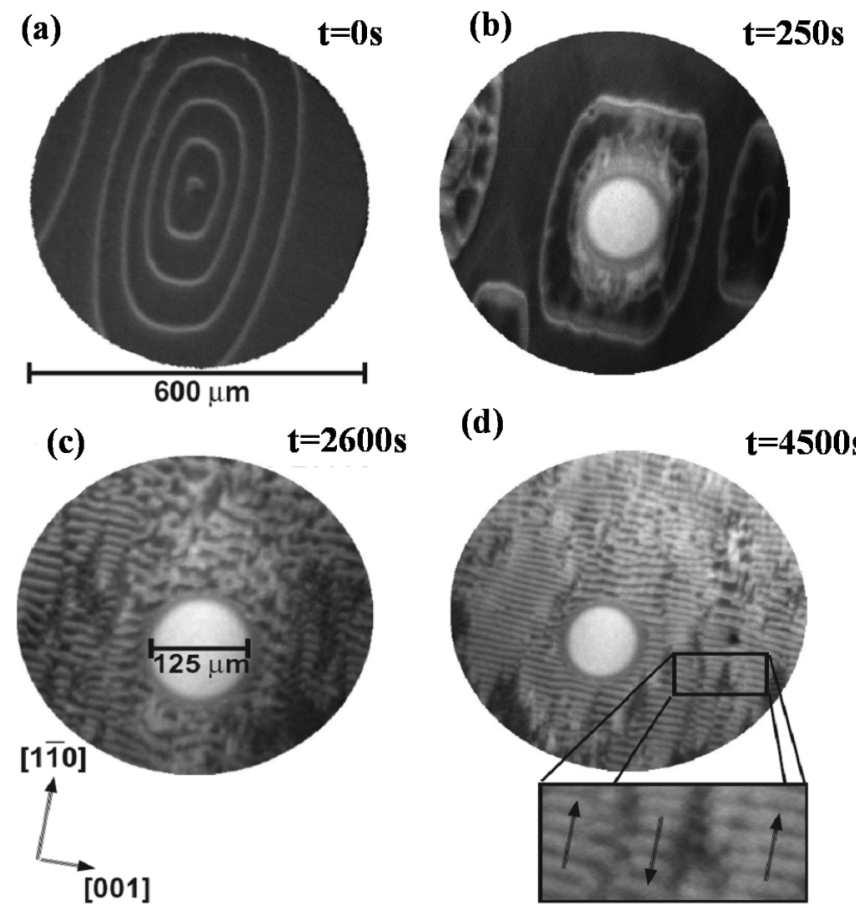

FIG. 1. Experimental observation of chemical patterns in the $\mathrm{NO}+\mathrm{H}_{2}$ reaction on a $\mathrm{Rh}(110)$ surface in the absence (a) and presence (b-d) of potassium as a promoter using photoelectron emission microscopy. (a) Typical target pattern in the absence of potassium. (b) Formation of large scale target patterns which are destabilized by short scale traveling waves upon a slight change in the experimental conditions (c,d). Adapted with permission from [31].

with experimentally observed patterns in the $\mathrm{NO}+\mathrm{H}_{2}$ reaction in the presence of potassium as a promoter [31].

We observe that two different mechanisms of pattern formation appear with different characteristic spatial scales. For weak molecular interactions the excitable dynamics of the system dominates and a local perturbation of the system leads to the rapid propagation of an excitation pulse. For strong molecular interactions short scale traveling waves dominate, which propagate more slowly than the pulses in the excitable regime. Similarly to the experiments, where the promotor changes the intensity of the molecular interactions, we can switch between both mechanisms changing the corresponding parameter of the model. Additionally we observe an interesting transition regime, where a short scale instability triggers periodically global excitation pulses, which resemble homogeneous oscillations.

The paper is organized as follows. We will first introduce a modified FitzHugh-Nagumo model with a transport term of the Cahn-Hilliard type for the activator species. Second we will perform a linear stability analysis of the uniform steady state complemented by full numerical simulations of the model in one dimension. Next, we perform anisotropic two-dimensional simulations to compare the patterns generated by this generic model with experimental patterns in the $\mathrm{NO}+\mathrm{H}_{2}$ reaction in catalytic surfaces [31]. Finally we discuss our results and conclude.

\section{MODEL}

We consider an abstract two-variable model of the FitzHugh-Nagumo type in two spatial dimensions in the excitable regime [1]. The model consists of a highly mobile activator species $u$ and an immobile inhibitor species $v$ with a slow kinetics:

$$
\begin{gathered}
\partial_{t} u=-u(u-a)(u-1)-v-\vec{\nabla} \cdot \vec{j}, \\
\partial_{t} v=\epsilon(u-b v) .
\end{gathered}
$$

In our model the parameters $a, b$, and $\epsilon \ll 1$ are positive constants. The activator species $u$ may diffuse on the surface according to a Cahn-Hilliard dynamics [3], which models attractive molecular interactions between the activator molecules. Consequently, the flux $\vec{j}$ in Eq. (1) can be derived from a symmetric Ginzburg-Landau energy functional:

$$
F[u]=\int_{\Omega} d A\left[\frac{1}{4} u^{4}-\frac{\tau}{2} u^{2}+\frac{\chi}{2}(\nabla u)^{2}\right],
$$

where $\tau$ and $\chi$ are positive constants. $\tau$ captures the local part of the molecular interactions and $\chi$ has the effect of a line tension and scales as $\chi \sim \tau r_{0}^{2}$, with $r_{0}$ being the typical length scale of an effective interaction potential, whereby $r_{0}$ is assumed to be large compared to the typical molecular scale [4]. This approach is in line with the experimental observations shown in Fig. 1, where the small scale wavelength caused by molecular interactions is of the order of $10 \mu \mathrm{m}$ and hence much larger than the lattice constants of the underlying surface, which are $<1 \mathrm{~nm}$. The first two terms in Eq. (3) represent a double well potential with a reflection symmetry about the concentration $u=0$ and with two minima located at $\pm \sqrt{\tau}$. The flux $\vec{j}$ of the species $u$ is then given by the gradient of the functional variation of $F$ with respect to $u$ [4] multiplied by a mobility D:

$$
\begin{aligned}
\vec{j} & =-\mathbf{D} \nabla \frac{\delta F}{\delta u} \\
& =-\mathbf{D} \nabla\left[u^{3}-\tau u-\chi \nabla^{2} u\right],
\end{aligned}
$$

where $\mathbf{D}$ is a tensor of second order of the form

$$
\mathbf{D}=\left(\begin{array}{cc}
D_{\|} & 0 \\
0 & D_{\perp}
\end{array}\right),
$$

with $D_{\|}>0$ and $D_{\perp}>0$. Such an anisotropy in the diffusion coefficients has been shown experimentally in chemical reactions on catalytic surfaces such as $\mathrm{Rh}(110)$ [39] and in this case it results from the rectangular symmetry of the surface lattice. For simplicity we assume a constant (i.e., density independent) mobility D. Note that the difference of Eqs. (1) and (2) from a standard FitzHugh-Nagumo model is only in the transport term of the activator species $u$, which is typically of the form $\vec{j}=-\mathbf{D} \nabla u$. Instead we introduce here a transport term of the Cahn- Hilliard type (5), which accounts for attractive molecular interactions between molecules of the activator species. 


\section{RESULTS}

Depending on the local parameters $a, b$, and $\epsilon$ the homogeneous system [Eqs. (1) and (2)] has up to three rest states and shows a variety of dynamical phenomena, e.g., oscillations and excitability. Here we restrict ourselves to the parameter region with just one uniform steady state $(\tilde{u}=0, \tilde{v}=0)$, and where the homogeneous system is excitable, i.e., the trivial rest state is linearly stable but if the system experiences a perturbation which exceeds the excitation threshold it will undertake a large excursion into the phase space before returning to its rest state. In a spatially extended system without molecular interactions a local excitation can lead to the propagation of an excitation pulse [see Fig. 4(a)].

In the absence of chemical reactions the transport term with attractive molecular interactions $[\tau>0$ in Eq. (5)] destabilizes the uniform state $(\tilde{u}=0)$ and induces the aggregation of the $u$ species into short scale stationary periodic domains with the initial spinodal wave number $k_{s}=\sqrt{\tau /(2 \chi)} \sim 1 / r_{0}$ that coarse in time due to line tension [4]. The saturation values of the $u$ species in the low and high density phase are given by $\pm \sqrt{\tau}$.

In the following we analyze the combined effect of local excitability and short scale spinodal decomposition. We fix the parameters of the local reaction kinetics to $b=3.0$ and $\epsilon=0.01$ and the parameter of the Cahn-Hilliard term to $\chi=$ 0.1 and we vary the chemical reaction parameter $a$ and the molecular interaction strength $\tau$.

\section{A. Linear stability analysis}

Applying Fourier mode perturbations with wave number $k$ and growth rate $\omega$ to the homogeneous trivial steady state we find for the Jacobian matrix $\mathbf{J}$ of Eqs. (1) and (2) for the case of an isotropic diffusion, i.e., $D_{\|}=D_{\perp}=D$,

$$
\mathbf{J}=\left(\begin{array}{cc}
-a+D k^{2}\left(\tau-\chi k^{2}\right) & -1 \\
\epsilon & -\epsilon b
\end{array}\right) .
$$

It is easy to see that the isotropic system displays a wave instability for

$$
\tau=\tau_{w}=2 \sqrt{\frac{\chi}{D}(a+\epsilon b)},
$$

with the critical wave number $k_{w}=\sqrt{\tau / 2 \chi}$ and the associated frequency $\omega_{w}=\sqrt{\epsilon\left(1-\epsilon b^{2}\right)}$. For $\tau>\tau_{w}\left(\tau<\tau_{w}\right)$ the trivial rest state is linearly unstable (stable). Interestingly, $k_{w}$ corresponds to the fastest growing mode $k_{s}$ of the spinodal decomposition of the Cahn-Hilliard term (5) in the absence of chemical reactions. For $\tau>\tau_{r}=2 \sqrt{\frac{\chi}{D}(a-\epsilon b+2 \sqrt{\epsilon})}$ unstable real eigenvalues are appearing, whereby they appear first at the critical wave number $k_{w}$.

To illustrate the type of linear instabilities encountered Fig. 2 shows examples of dispersion relations for various sets of parameters. Figures 2(a) and 2(b) show the dispersion relation at the wave instability and for parameters, where all unstable eigenvalues are complex, whereas Figs. 2(c) and 2(d) show the dispersion relation at the point where real unstable eigenvalues first appear and for parameters where the fastest growing eigenvalues are real. Additionally, the dashed and

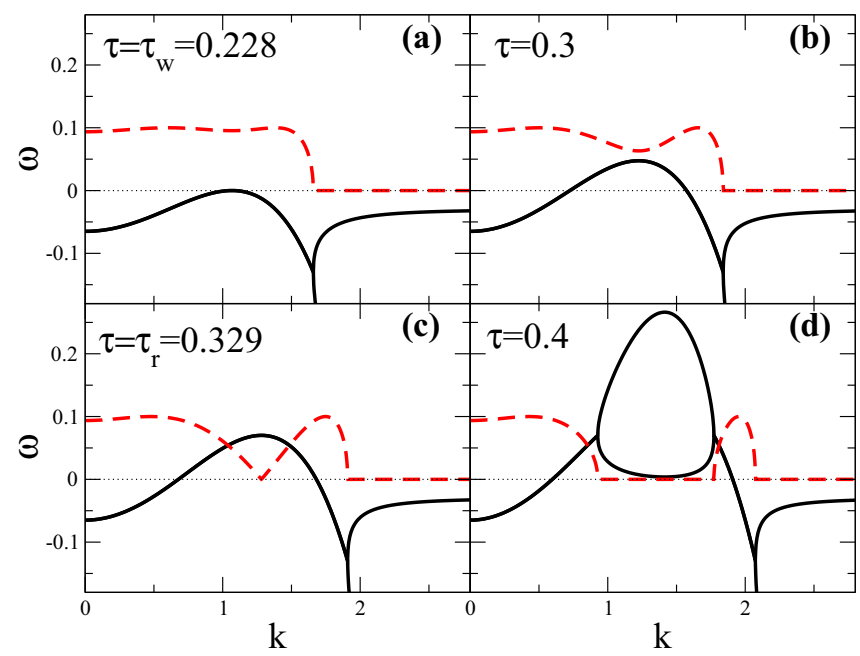

FIG. 2. (Color online) Dispersion relations of Eqs. (1) and (2) for various values of $\tau$ as indicated in the top left corner of each graph. The solid (dashed) lines denote the real (imaginary) parts of the two eigenvalues. Remaining parameters are $a=0.1, b=3.0, \varepsilon=0.01$, $D=D_{\|}=D_{\perp}=1$, and $\chi=0.1$.

dotted lines in Fig. 3 show, respectively, the wave instability [Fig. 2(a)] and the first appearance of real unstable eigenvalues [Fig. 2(c)] in the $a-\tau$ plane.

\section{B. One-dimensional numerical simulations}

To study the nonlinear evolution of the system we performed numerical simulations of Eqs. (1) and (2) in one spatial dimension with a periodic boundary condition using a finite differences method. Simulations were started from two different initial conditions. For one type of simulation we started out from the homogeneous steady state with small amplitude white noise perturbations. For the second type of simulation we started from the homogeneous rest state with a local perturbation, which was sufficient to locally excite the

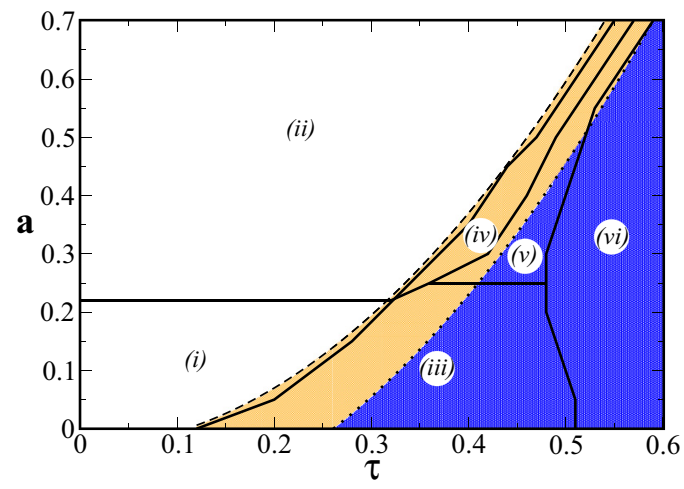

FIG. 3. (Color online) Phase diagram of full numerical simulations in one dimension of (1-2) in the $a-\tau$ plane. The regions are (i) excitable, (ii) nonexcitable, (iii) global oscillations, (iv) standing waves, (v) a mix of standing and traveling waves, and (vi) traveling waves. The dashed and dotted lines indicate the wave instability and the appearance of unstable real eigenvalues from the linear stability analysis. Remaining parameters are as in Fig. 2. 


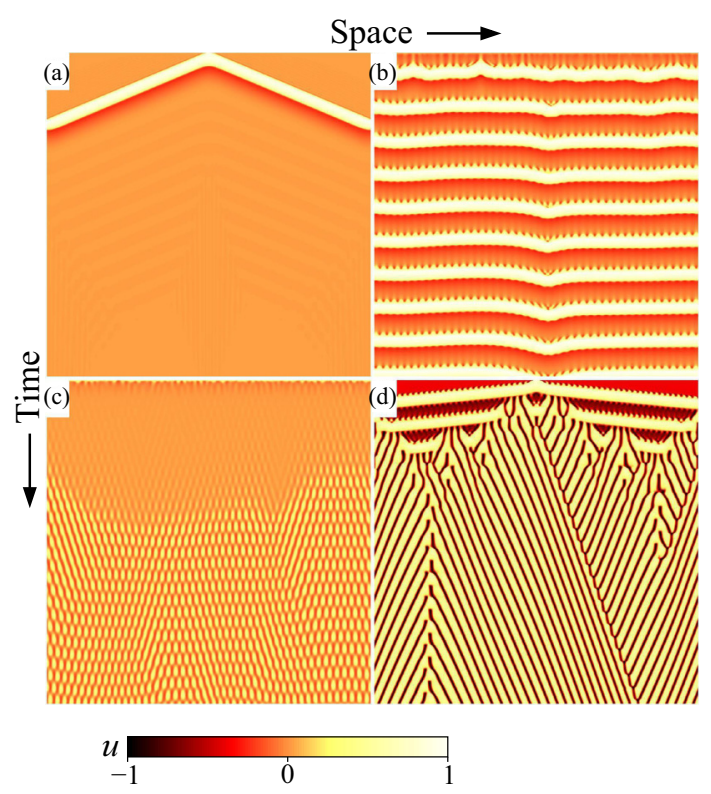

FIG. 4. (Color online) Spatiotemporal plots of the variable $u$ for one-dimensional simulations for different values of $a$ and $\tau: a=0.1$, $\tau=0.1$ (a); $a=0.1, \tau=0.4$ (b); $a=0.5, \tau=0.5$ (c); and $a=0.1$, $\tau=0.6$ (d). Simulations were started from the trivial rest state with either small amplitude random perturbations $(b, c)$ or a finite localized perturbation (a,d). The domain size is 200 with periodic boundary conditions and the simulation time is 1000 . Remaining parameters are as in Fig. 2.

system and trigger the propagation of an excitation pulse in the absence of molecular interactions (i.e., $\tau=0$ ). The localized perturbation permits us to differentiate between the excitable and nonexcitable regimes of the model.

Figure 3 (solid lines) shows the resulting phase diagram in the $a-\tau$ plane along with the results of the linear stability analysis [Fig. 3 (dashed and dotted lines)]. As an illustration Fig. 4 shows examples of simulations for qualitatively different cases. In regions (i) and (ii) of Fig. 3 the system is linearly stable but in region (i) it permits the propagation of excitation pulses, i.e., the local perturbation in the initial condition triggers a pulse, which is annihilated due to the periodic boundary conditions [Fig. 4(a)]. In region (iii) in Fig. 3, i.e., for parameter values close to the wave instability and the excitable region (i), a short scale stationary instability driven by the Cahn-Hilliard term (5) is triggering an excitation pulse. Since the stationary domains are each triggering a pulse almost simultaneously, the excitation is nearly global. After the excitation pulse has decayed the system enters into a recovery phase and appears to be homogeneous until new short scale stationary domains form, which trigger in turn excitation pulses. The resulting dynamics resembles an oscillatory behavior [Fig. 4(b)]. In region (iv) of Fig. 3, i.e., close to the wave instability and outside the excitable region, one can observe short scale standing waves [Fig. 4(c)], whereas in region $(v)$ further away from the wave instability the system shows a mix of short scale traveling waves and standing waves. Finally, in region (vi) of Fig. 3 the system shows short scale traveling waves [Fig. 4(d)]. Thereby the traveling waves move

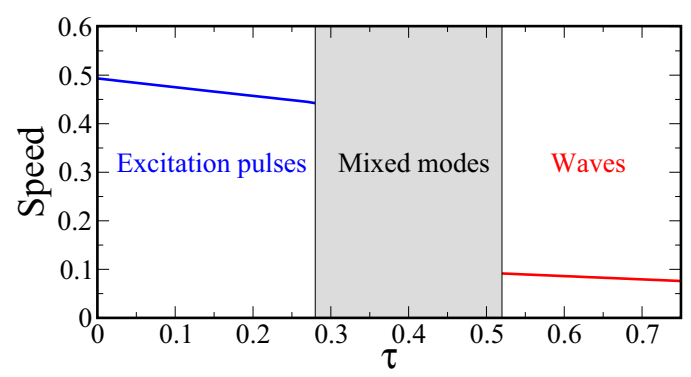

FIG. 5. (Color online) Wave velocity depending on the molecular interaction strength $\tau$ at a constant value $a=0.1$. Shown is the velocity for small and large $\tau$. In the region denoted "Mixed modes" several patterns coexist and the wave velocity is not clearly defined. Remaining parameters are as in Fig. 2.

at a much slower speed than the excitation pulses in the excitable regime.

To quantify the slowdown of the wave speed Fig. 5 shows the dependence of the wave speed on the strength of the molecular interactions $\tau$ for a constant parameter $a=0.1$. For small $\tau$ large scale excitation pulses are stable and propagate at a high velocity, which decreases linearly with $\tau$. At intermediate values of $\tau$ in the region denoted "Mixed modes" in Fig. 5 the system exhibits an oscillatory behavior of short scale destabilization and global pulses described above. For high values of $\tau$, short scale traveling waves are stable and travel slowly, whereby the speed decreases linearly with $\tau$ and is about four times slower than the speed of the excitation pulses.

\section{Two-dimensional numerical simulations}

For a better comparison with the experiments on the catalytic $\mathrm{NO}+\mathrm{H}_{2}$ reaction on a $\mathrm{Rh}(110)$ surface [31] we performed two-dimensional full numerical simulations of Eqs. (1) and (2) with periodic boundary conditions using a pseudospectral method. In one set of simulations we used isotropic mobility coefficients $D=D_{\|}=D_{\perp}=1.0$ and in a second set we assumed anisotropic mobility coefficients with $D_{\|}=1.0$ and $D_{\perp}=4.0$.

Figure 6 shows snapshots of two numerical simulations which were started from the uniform rest state with small amplitude white noise. The left and right columns correspond to the isotropic and anisotropic case, respectively. The parameters were chosen from the region (vi) of Fig. 3 where short scale traveling waves can be found in one-dimensional systems. In the isotropic case small scale traveling waves with an initially irregular structure develop, which move in small wave packets into different directions. Upon collision, waves are annihilated. As time proceeds the size of wave packets increases and the waves become more regular. However, on the time scale of simulation we did not observe the motion of coherent waves into one direction. More extensive simulations are necessary to identify such a spontaneous symmetry breaking. In the anisotropic case the initially irregular waves rapidly evolve into almost plane waves which move in the direction of the fast mobility constant, whereby regions of opposite propagation direction coexist. 


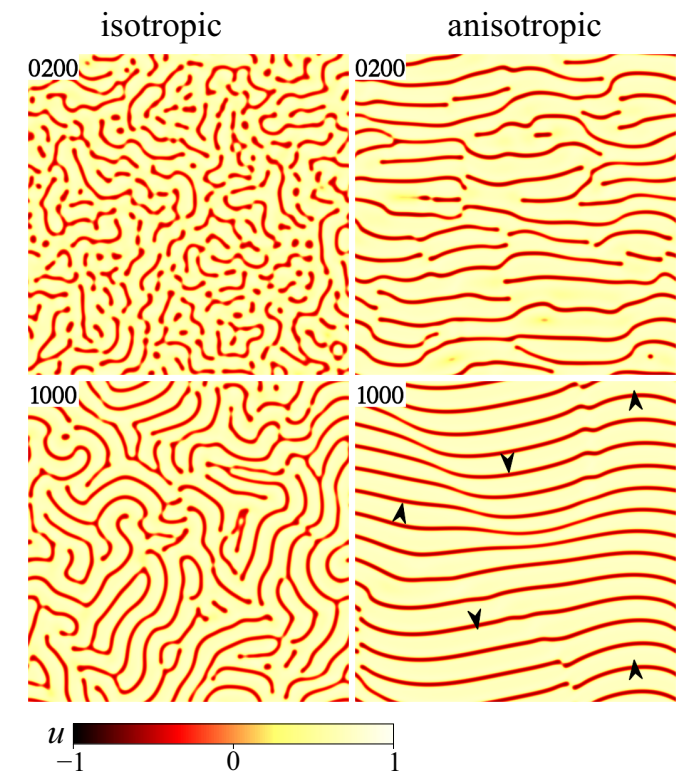

FIG. 6. (Color online) Snapshots of two-dimensional numerical simulations with isotropic (left) and anisotropic mobility (right) starting from the trivial rest state with small amplitude perturbations. Shown is the spatial distribution of the activator concentration $u$. The black arrow heads indicate the direction of propagation of the traveling wave packets. Parameters are $a=0.1, \tau=0.6, D_{\|}=1.0$, $D_{\perp}=1.0$ (left), $D_{\perp}=4.0$ (right), and as indicated in Fig. 2. The simulation time is indicated in the top left corner of each snapshot. The domain size is $128 \times 128$ with periodic boundary conditions.

Figure 7 shows the results of a simulation with anisotropic mobility constants in the presence of a pacemaker. The pacemaker consisted of a small region in the center of the simulation domain where the concentrations of the $u$ and $v$ field were kept constant at $u=1.0$ and $v=0.0$ during the integration. We started the simulation for a parameter set where the system is locally excitable, i.e., $a=0.1$ and $\tau=0$, and the pacemaker triggered a target pattern which was elongated in the direction of the large mobility constant (see snapshot labeled 500 in Fig. 7). After the pacemaker had triggered several pulses we increased the parameter $\tau$ to $\tau=0.6$ at the time $t=500$ and observed a rapid decay of the large scale waves into short scale traveling waves (see snapshots labeled 510 to 2000 in Fig. 7), which traveled into the direction of the large mobility constant. Thereby regions of opposite propagation direction coexisted over a long time. Immediately after an increase in the parameter $\tau(500<t<550)$ a short scale instability developed, which competed with the target pattern. At later stages the pacemaker completely lost control over the pattern and the short scale traveling waves took over. The transition from target pattern to small scale traveling waves is nicely illustrated in the space-time plot (Fig. 7 bottom) along a vertical line through the center of the simulation domain.

The breakup of the target pattern into small scale parallel wave trains resembles the experimentally observed breakup of a target pattern in the $\mathrm{NO}+\mathrm{H}_{2}$ reaction on a $\mathrm{Rh}(110)$ surface upon addition of potassium (see Fig. 1). There, the anisotropy of the surfaces privileges the formation of wave trains of varying size which travel along the direction of fast
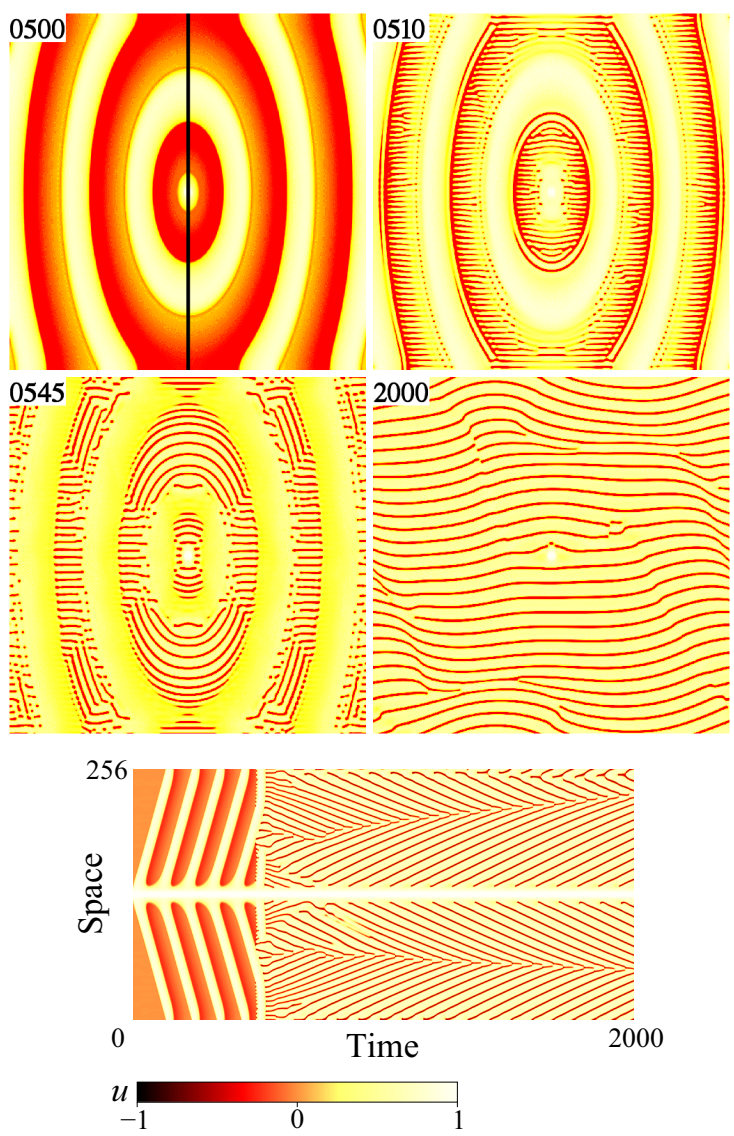

FIG. 7. (Color online) Two-dimensional simulation with anisotropic mobility coefficients in the presence of a pacemaker in the center of the computational domain. Shown is the concentration of the $u$ field. Two top rows: Snapshots with the simulation time indicated in the top left corner. Bottom: Space-time plot for a vertical line through the center of the simulation domain as indicated by the black line for the snapshot at $t=500$. For a simulation time $t<500$ the parameter $\tau=0$ and for $t>500$ the parameter $\tau=0.6$. Simulations were started from the trivial rest state with small amplitude white noise. The domain size is $256 \times 256$ with periodic boundary conditions. The remaining parameters are $D_{\|}=1.0$, $D_{\perp}=4.0$, and as indicated in Fig. 2 .

diffusion, identified by the long axis of the elliptic target pattern. Furthermore, the simulations satisfactorily reproduce the difference in the wave velocity between both regimes.

\section{DISCUSSION}

We have employed a FitzHugh-Nagumo model with a transport term of the Cahn-Hilliard type to analyze the dynamics of an excitable system with molecular interactions. The linear stability analysis of the homogeneous rest state revealed an oscillatory short wavelength instability, whose properties depend on the strength of the molecular interactions. Numerical simulations of the system reveal a rich spatiotemporal behavior. In the parameter region where the system is locally excitable and close to the wave instability we find an interesting transition regime where a short scale instability is triggering a globally oscillatory behavior. Further away from the wave instability and for parameters where excitation pulses 
do not propagate, the system displays standing or traveling waves on a small spatial scale, with typical wave speeds much slower than the speed of excitation pulses. Numerical simulations in two dimensions with an anisotropy in the mobility of the activator species and in the presence of a pacemaker displayed the same transition from large scale target patterns to small scale traveling waves upon an increase in the molecular interactions, as seen experimentally in the $\mathrm{NO}+\mathrm{H}_{2}$ reaction on a $\mathrm{Rh}(110)$ surface upon the addition of potassium (see Figs. 1 and 7 right column). Furthermore our results agree qualitatively with the experimentally observed slowing down of the wave speed upon the addition of the promotor.

The occurrence of a wave instability in reaction-diffusion systems with molecular interactions has been shown before for a variety of models, e.g., [13,14], and seems to be a rather general feature in these systems. The difference arises from the detailed dynamics of the considered system and the degree of nonlinearities retained in the final equations. In [13] a chemical surface reaction with adsorption and desorption is studied. In this system molecular interactions modify the transport as well as the desorption kinetics of one species and lead to a wave bifurcation in the vicinity of a two-phase coexistence region. Reference [14] considers a hypothetic cyclic linear chemical reaction coupled to a Cahn-Hilliard term, where the molecular interactions trigger a wave bifurcation from a stable homogeneous state. In contrast we have studied here a situation where molecular interactions interact with an excitable dynamics and cause a wave instability.

The transition regime close to the wave instability, where molecular interactions trigger excitation pulses, offers inter- esting perspectives for understanding signaling mechanisms in cell biological systems. In the plasma membrane of living cells proteins and lipids are in close proximity and molecular interactions compete with reaction-diffusion mechanisms $[17,40]$. It is possible that a local event leads to the clustering of molecules due to molecular interactions, which then could trigger an excitation pulse that travels over the whole cell membrane.

In summary, the analysis of a generic activator-inhibitor model with short-range molecular interactions (short compared to the typical reaction-diffusion length scale) shows the formation of two types of patterns. While large scale structures are reproduced by a typical reaction-diffusion mechanism, small scale patterns are the consequence of the molecular interactions. Depending on the control parameters, both mechanisms compete and produce new dynamics. The results of the model show good qualitative agreement with the patterns obtained in the $\mathrm{NO}+\mathrm{H}_{2}$ reaction on a $\mathrm{Rh}(110)$ surface.

\section{ACKNOWLEDGMENTS}

We thank Prof. Imbihl for discussions and for providing us with Fig. 1. S.A. and M.B. acknowledge financial support from the German Science Foundation (Deutsche Forschungsgemeinschaft) within the framework of Grant No. SFB 910 (Control of Self-Organizing Nonlinear Systems). The LIPhy is part of the LabEx Tec 21 (Investissements de l'Avenir Grant No. ANR-11-LABX-0030).
[1] Chemical Waves and Patterns, edited by R. Kapral and K. Showalter (Kluwer, Dordrecht, 1994).

[2] J. Keener and J. Sneyd, Mathematical Physiology (Springer, New York, 2009), Vol. 8.

[3] J. W. Cahn and J. E. Hilliard, J. Chem. Phys. 28, 258 (1958).

[4] C. Godrèche, Solids Far from Equilibrium (Cambridge University Press, Cambridge, 1991), Vol. 1.

[5] S. C. Glotzer, E. A. Di Marzio, and M. Muthukumar, Phys. Rev. Lett. 74, 2034 (1995).

[6] J. Verdasca, P. Borckmans, and G. Dewel, Phys. Rev. E 52, R4616 (1995).

[7] H. P. Bonzel, A. M. Bradshaw, and G. Ertl, in Material Science Monographs (Elsevier, Amsterdam, 1989), Vol. 67.

[8] M. P. Kiskinova, Poisoning and Promotion in Catalysis Based on Surface Science Methods, Studies in Surface Science and Catalysis Vol. 70 (Elsevier, Amsterdam, 1991).

[9] H. Marbach, M. Hinz, S. Günther, L. Gregoratti, M. Kiskinova, and R. Imbihl, Chem. Phys. Lett. 364, 207 (2002).

[10] Y. D. Decker, H. Marbach, M. Hinz, S. Günther, M. Kiskinova, A. S. Mikhailov, and R. Imbihl, Phys. Rev. Lett. 92, 198305 (2004).

[11] M. Hinz, S. Günther, H. Marbach, and R. Imbihl, J. Phys. Chem. B 108, 14620 (2004).

[12] Y. DeDecker and A. S. Mikhailov, J. Phys. Chem. B 108, 14759 (2004).
[13] M. Hildebrand, A. S. Mikhailov, and G. Ertl, Phys. Rev. Lett. 81, 2602 (1998).

[14] T. Okuzono and T. Ohta, Phys. Rev. E 67, 056211 (2003).

[15] J. Verdasca and P. Borckmans, G. Dewel, Phys. Rev. E 64, 055202(R) (2001).

[16] M. Hildebrand, M. Ipsen, A. S. Mikhailov, and G. Ertl, New J. Phys. 5, 61 (2003).

[17] A. S. Mikhailov and G. Ertl, Chem. Phys. Chem. 10, 86 (2009).

[18] R. Reigada, F. Sagués, and A. S. Mikhailov, Phys. Rev. Lett. 89, 038301 (2002).

[19] T. Okuzono, Y. Tabe, and H. Yokoyama, Phys. Rev. E 69, 050701 (2004).

[20] T. Shibata and A. S. Mikhailov, Europhys. Lett. 73, 436 (2006).

[21] T. Shibata and A. S. Mikhailov, Chaos: An Interdisciplinary Journal of Nonlinear Science 16, 037108 (2006).

[22] E. L. Elson, E. Fried, J. Dolbow, and E. G. Genin, Annu. Rev. Biophys. 39, 207 (2010).

[23] K. Kruse, Biophys. J. 82, 618 (2002).

[24] K. John and M. Bär, Phys. Rev. Lett. 95, 198101 (2005).

[25] K. John and M. Bär, Phys. Biol. 2, 123 (2005).

[26] G. Meacci and K. Kruse, Phys. Biol. 2, 89 (2005).

[27] S. Alonso and M. Bär, Phys. Biol. 7, 046012 (2010).

[28] Y. Arai, T. Shibata, and S. Matsuoka, Proc. Natl. Acad. Sci. USA 107, 12399 (2010).

[29] N. W. Goehring, P. K. Trong, J. S. Bois, D. Chowdhury, E. M. Nicola, A. A. Hyman, and S. W. Grill, Science 334, 1137 (2011). 
[30] M. Bonny, E. Fischer-Friedrich, M. Loose, P. Schwille, and K. Kruse, PLoS Comp. Biol. 9, e1003347 (2013).

[31] H. Marbach, S. Günther, T. Neubrand, and R. Imbihl, Chem. Phys. Lett. 395, 64 (2004).

[32] G. Ertl, Science 254, 1750 (1991).

[33] F. Mertens and R. Imbihl, Nature (London) 370, 124 (1994).

[34] H. H. Rotermund, G. Haas, R. U. Franz, R. M. Tromp, and G. Ertl, Science 270, 608 (1995).

[35] A. Schaak and R. Imbihl, J. Chem. Phys. 107, 4741 (1997).
[36] A. Makeev, M. Hinz, and R. Imbihl, J. Chem. Phys. 114, 9083 (2001).

[37] L. Hong, H. Uecker, M. Hinz, L. Qiao, I. G. Kevrekidis, S. Günther, T. O. Mentes, A. Locatelli, and R. Imbihl, Phys. Rev. E 78, 055203 (2008).

[38] L. Hong, M. Hesse, and R. Imbihl, J. Phys. Chem. C 113, 4174 (2009).

[39] M. Kiskinova, Chem. Rev. 96, 1431 (1996).

[40] T. Misteli, J. Cell Biol. 155, 181 (2001). 